\title{
Clinical characteristics of adverse reactions to nonionic low osmolality contrast media in patients transferred from the CT room to the emergency room
}

\author{
Sang Ook Ha${ }^{1}$, Dae Yong Kim² and You Dong Sohn ${ }^{\text {* }}$
}

\begin{abstract}
Nonionic low osmolality contrast media (LOCMs) are used universally in computed tomography (CT) imaging. Although adverse reactions due to nonionic LOCMs are a common cause of emergency room (ER) admissions, few studies have investigated these adverse reactions. In the present study, we evaluated the characteristics of patients who were transferred from the CT room to the ER due to adverse reactions to contrast media, and we determined the risk factors for severe adverse reactions. A single-center retrospective study was conducted over a 41-month period. Baseline and clinical characteristics were evaluated and analyzed according to moderate and severe severity. In particular, risk factors of severe reactions were determined using logistic regression analysis. In total, 70 patients were admitted to the ER with adverse reactions due to nonionic LOCMs. Of these, 33 developed a moderate reaction, and 37 developed a severe reaction. Compared with the moderate reaction group, the severe reaction group was older, had higher blood pressures, showed more symptoms indicating the cardiovascular and central nervous system, and developed faster reactions to LOCMs. According to the multivariate logistic regression analysis, the age of the patient and time to onset of reaction demonstrated a statistical relationship with severe adverse reactions. In the receiver operating characteristic analysis, the optimal cutoff values for age and time to onset were 60 years and $5 \mathrm{~min}$. In conclusion, clinicians should be attentive to anaphylaxis due to nonionic LOCM, in particular, for elderly patients aged older than 60 years and a time to reaction onset of less than 5 min.
\end{abstract}

Keywords: Adverse reaction, Contrast media, Severity

\section{Background}

Contrast media are currently used for imaging at least one to 70 million times per year in the United States (Christiansen 2005). Computed tomography (CT) examination with administration of contrast media causes a risk of developing adverse reactions to the contrast media. Compared with the use of ionic contrast media, nonionic low osmolality contrast media (LOCMs) have been associated with a reduced overall prevalence of

\footnotetext{
*Correspondence: medysohn@hallym.or.kr

${ }^{1}$ Department of Emergency Medicine, Hallym University Sacred Heart Hospital, Hallym University Medical Center, 22, Gwanpyeong-ro 170 Beon-gil, Dongan-gu, Anyang-si, Gyeonggi-do 431-070, Korea

Full list of author information is available at the end of the article
}

adverse reactions (Grant and Camamo 1997; Jacobs et al. 1998). However, adverse reactions due to nonionic LOCMs remain an important cause of admissions to the emergency room (ER). Furthermore, the lower incidence of adverse reactions resulted in fewer opportunities to develop and maintain these necessary skills (Segal and Bush 2011). In the present study, we evaluated the characteristics of patients transferred from the CT room to the ER with moderate or severe adverse reactions due to nonionic LOCMs and evaluated the risk factors for severe adverse reactions. 


\section{Methods}

\section{Study design and patient selection}

This study was approved by the author's Institutional Review Board for the evaluation and analysis of patient data. Due to the purely observational, retrospective, and non-interventional nature of this study, informed consent was deemed unnecessary and was not obtained. Patient records and information were anonymized and de-identified prior to analysis. Our protocol for adverse reactions to contrast media was as follows: (1) patients with moderate or severe reactions to contrast media received an injection [intravenous antihistamine $(4 \mathrm{mg})$ and methylprednisolone $(0.5 \mathrm{mg} / \mathrm{kg})$ with or without intramuscular epinephrine $(0.3 \mathrm{mg})$ ] and were transferred from the CT room to the ER. (2) Patients with mild reactions were discharged (with or without antihistamine administration) from the CT room. From January 2010 to May 2013, patients with moderate or severe reactions to contrast media who were transferred from the CT room to the ER were enrolled in the present study. For the inclusion of patients with delayed moderate or severe adverse reactions, the diagnostic terms 'anaphylaxis', 'anaphylactic shock', and 'anaphylactic reaction' provided by the "International Statistical Classification of Diseases and Health-related Problems, 10th revision (ICD 10)" were used to identify the patients. Among various causes, only contrast media were included in the present study. Patients aged younger than 18 years were excluded from the analysis.

\section{Data collection}

Baseline characteristics, including gender; age; vital signs; history of allergy to food, drugs, or contrast agents; morbidity; history of medication; and clinical characteristics, including type of contrast medium, premedication, treatments, symptoms and disposition, were collected as data in the electronic medical records. The records for time to onset of adverse reaction, immediate symptoms and vital signs were reviewed in report form written by the radiology department for moderate or severe adverse reactions according to the hospital policy.

\section{Comparison of moderate and severe adverse reactions}

Patients were grouped into moderate and severe groups according to the severity of the adverse reactions they developed in response to contrast media. The baseline and clinical characteristics of the patients were compared by group. Risk factors for severe reactions were determined using univariate and subsequent multivariate logistic regression analyses. A receiver operating characteristic (ROC) analysis was performed to determine the optimal cutoff values for each potential risk factor.

\section{Definition and classification of adverse and immediate reactions to contrast media}

In the recently published "2013 ACR Manual on Contrast Media-Version 9", adverse reactions are categorized as allergic-like reactions and physiologic reactions (ACR Committee on Drugs and Contrast Media 2013). However, when the reaction was severe, there were no significant differences in clinical manifestations and treatment methods between the two reaction categories. Therefore, the severity of the adverse reactions for this particular study was classified according to the "ACR Manual on Contrast Media-Version 8", as shown in Table 1 (ACR Committee on Drugs and Contrast Media 2012). A reaction was identified as immediate if it occurred within the first hour of administration of LOCM and as delayed if it occurred after the first hour had passed.

\section{Selection of contrast media and premedication protocol}

Throughout the study, all contrast media used in our center were nonionic LOCMs. The dose, rate, and type of contrast media were determined according to the protocol provided by our center. Contrast media (100-150 mL) were administered at a rate of $2.5-4 \mathrm{~mL} / \mathrm{s}$. If a patient had previously developed a moderate or severe reaction to a certain contrast medium, other contrast agents were recommended for the next administration, and premedication [intravenous Peniramin $(4 \mathrm{mg}$ at $1 \mathrm{~h}$ ) and Cortisol (200 $\mathrm{mg}$ at 4 and $1 \mathrm{~h}$ ) prior to contrast administration] were used.

\section{Statistical analysis}

The normality of the data distribution was evaluated using the Kolmogorov-Smirnov test, and the statistical methods used for analysis were selected accordingly. Median values (with 25-75th percentile values in parentheses) were used to present continuous data, which were not normally distributed. Numerical values (with percentile values in parentheses) were used to show categorical data. Categorical data were analyzed using the Chi squared or Fisher exact tests, whereas continuous data were analyzed using the Mann-Whitney $U$ test for two-group unpaired comparisons. Risk factors for severe reactions were determined using multivariate logistic regression analysis, which included all variables that provided a $p$ value $\leq 0.2$ in the univariate analysis. Subsequently, a ROC analysis was performed to determine the optimal cutoff values for each potential risk factor. For all comparisons, tests were two-tailed, and $p$ values $<0.05$ were regarded as statistically significant. SPSS 18.0.0 software for Windows (SPSS, Inc., Chicago, IL) was used for all statistical analyses. 
Table 1 Classification of adverse reactions in response to contrast media

\begin{tabular}{|c|c|c|c|}
\hline Severity & Categories of reactions & & \\
\hline Mild & $\begin{array}{l}\text { Nausea, vomiting } \\
\text { Cough } \\
\text { Warmth } \\
\text { Headache } \\
\text { Dizziness } \\
\text { Tremor }\end{array}$ & $\begin{array}{l}\text { Altered taste } \\
\text { Itchiness } \\
\text { Pallor } \\
\text { Flushing } \\
\text { Chills }\end{array}$ & $\begin{array}{l}\text { Perspiration } \\
\text { Rash, hives } \\
\text { Nasal stuffiness } \\
\text { Swelling of the eyes and face } \\
\text { Anxiety }\end{array}$ \\
\hline Moderate & $\begin{array}{l}\text { Tachycardia } \\
\text { Bradycardia } \\
\text { Hypertension } \\
\text { Generalized or diffuse erythema }\end{array}$ & $\begin{array}{l}\text { Dyspnea } \\
\text { Bronchospasm } \\
\text { Wheezing }\end{array}$ & $\begin{array}{l}\text { Laryngeal edema } \\
\text { Mild hypotension }\end{array}$ \\
\hline Severe & $\begin{array}{l}\text { Laryngeal edema (severe or rapidly progressing) } \\
\text { Unresponsiveness }\end{array}$ & $\begin{array}{l}\text { Cardiopulmonary arrest } \\
\text { Convulsions }\end{array}$ & $\begin{array}{l}\text { Profound hypotension } \\
\text { Clinically manifested arrhythmia }\end{array}$ \\
\hline
\end{tabular}

\section{Results}

\section{Baseline characteristics}

The baseline characteristics of the patients enrolled in the study are summarized in Table 2. Patients in the severe group were older (55 vs. $59, p=0.068$ ) and had lower blood pressures $(110 / 69$ vs. $72 / 41, p<0.001)$ compared with those in the moderate group. Between the two groups, there were no significant differences in gender; heart rate; respiratory rate; history of allergy to foods, drugs, or contrast media; morbidity due to hypertension, diabetes mellitus, or asthma; or history of medication. The most widely administered LOCM was Ultravist (34 times); Iopamiro and Xenetix were used infrequently (2 times).

\section{Time to onset of reaction and symptoms}

The time to onset of reaction was significantly different between the moderate and severe groups (7.0 vs. $4.0 \mathrm{~s}$, $p<0.001)$. The incidence of an immediate reaction that occurred within the first hour was significantly higher for the severe group compared with the moderate group (81.8 vs. $100 \%, p=0.008)$.

The most common symptom was skin-related or mucosal (urticarial and angioedema), followed in decreasing order by cardiovascular, respiratory, central nervous and gastrointestinal system-related symptoms. Relative to the moderate reactions, severe reactions more frequently involved cardiovascular and central nervous system symptoms, as shown in Table 3 (27.3 vs. $67.6 \%$, $p<0.001$, and 24.2 vs. $97.3 \%, p=0.001)$.

\section{Premedication, treatment, and outcome}

More patients in the severe group received premedication (48.6\%) than those in the moderate group (30.3\%). However, among the pretreated patients, there were no significant differences in the number of adverse reactions between the two groups, as shown in Table 4. To treat adverse reactions, an $\mathrm{H}_{1}$ blocker (67 cases) was used most frequently, followed by epinephrine (48 cases), an $\mathrm{H}_{2}$ blocker (46 cases), hydrocortisone (38 cases), methylprednisolone (32 cases), and a $\beta_{2}$ agonist nebulizer (4 cases). Excluding epinephrine, there were no significant differences in the number of medications used for treatment between the two groups. Epinephrine was more widely used to treat patients in the severe group compared with the moderate group (100 vs. $33.3 \%$, $p<0.001)$. More patients with severe reactions were admitted to the hospital than those with moderate reactions ( 45.9 vs. $12.1 \%, p=0.004)$. There were no mortalities in either group.

\section{Risk factor of severe adverse reactions}

Multivariate logistic regression analysis revealed that, after adjusting for previous adverse events to LOCMs, change in contrast, and pretreatment, the age of the patient and time to onset of the reaction were statistically significant factors for the development of severe reactions, as shown in Table 5 [odds ratio (OR) 1.053, $p=0.042$, and OR $0.805, p=0.020$ ]. The clinical relevance of these risk factors was further confirmed through subsequent ROC analysis. The areas under the ROC curves were 0.627 [95\% confidence interval (CI) 0.497-0.757, $p=0.068$ ] for patient age and 0.756 (95\% CI $0.639-0.873, p<0.001)$ for time to onset of reaction. The optimal cutoff values for age and time to onset of reaction for severe reactions was 60 years (sensitivity of $43.2 \%$ and specificity of $75.8 \%$ ) and 5 min (sensitivity of $72.7 \%$ and specificity of $70.3 \%$ ), respectively (data not shown).

\section{Discussion}

Use of nonionic LOCMs and enforcement of pretreatment have been proposed as methods to minimize adverse reactions in response to contrast media. Nevertheless, attention must be given to adverse reactions resulting from the use of nonionic LOCMs. If a comprehensive guideline regarding prevention and treatment is 
Table 2 Baseline characteristics of the enrolled patients

\begin{tabular}{|c|c|c|c|}
\hline Variables & Moderate $(n=33)$ & Severe $(n=37)$ & $p$ \\
\hline Sex (M:F ratio) & $17: 16$ & 18:19 & 0.811 \\
\hline Age (year) & $55(45-61)$ & $59(51-72)$ & 0.068 \\
\hline \multicolumn{4}{|l|}{ Initial vital signs } \\
\hline $\mathrm{SBP}(\mathrm{mmHg})$ & $110(104-120)$ & $69(60-80)$ & $<0.001$ \\
\hline $\mathrm{DBP}(\mathrm{mmHg})$ & $72(70-80)$ & $41(35-50)$ & $<0.001$ \\
\hline Heart rate (beats/min) & $86(68-96)$ & $75(71-89)$ & 0.708 \\
\hline $\begin{array}{l}\text { Respiratory rate } \\
\text { (breaths/min) }\end{array}$ & $20(18-23)$ & $16(14-18)$ & 0.400 \\
\hline Previous allergy & $15(45.5)$ & $16(43.2)$ & 0.853 \\
\hline Drug & & & 0.600 \\
\hline Pyrin & 1 & 1 & \\
\hline Hydrocortisone & 0 & 2 & \\
\hline NSAID & 1 & 0 & \\
\hline Food & & & 0.599 \\
\hline Seafood & 1 & 0 & \\
\hline Pupa & 1 & 0 & \\
\hline Mushroom & 0 & 1 & \\
\hline CT contrast media & $12(36.4)$ & $20(54.1)$ & 0.138 \\
\hline \multicolumn{4}{|l|}{ Morbidity } \\
\hline Hypertension & $13(39.4)$ & $10(17.0)$ & 0.271 \\
\hline Diabetes mellitus & $5(15.2)$ & $3(8.1)$ & 0.462 \\
\hline Asthma & 0 & 0 & \\
\hline \multicolumn{4}{|c|}{ Concurrent medications } \\
\hline \multicolumn{2}{|c|}{ Calcium channel blocker8 (24.2) } & $8(21.6)$ & 0.794 \\
\hline ACE inhibitor & $8(24.2)$ & $4(10.8)$ & 0.135 \\
\hline Beta blocker & $3(9.1)$ & $2(5.4)$ & 0.661 \\
\hline Type of contrast media & & & 0.436 \\
\hline lomeron & 3 & 2 & \\
\hline lopamiro & 2 & 0 & \\
\hline Omnipaque & 3 & 1 & \\
\hline Optiray & 2 & 5 & \\
\hline Pamiray & 8 & 7 & \\
\hline Ultravist & 14 & 21 & \\
\hline Xenetix & 1 & 1 & \\
\hline Contrast change & $23(69.7)$ & $20(54.1)$ & 0.180 \\
\hline
\end{tabular}

$A C E$ angiotensin converting enzyme, $C T$ computed tomography, DBP diastolic blood pressure, $F$ female, $M$ male, NSAID non-steroid anti-inflammatory drug, $S B P$ systolic blood pressure

not available, such reactions can be life threatening or fatal. In the present study, the baseline and clinical characteristics of patients who developed moderate or severe reactions to contrast media and were transferred from the CT room to the ER were evaluated and compared between moderate and severe groups. Compared with patients with a moderate reaction, those with a severe reaction were older, had higher blood pressures, showed more symptoms indicative of cardiovascular and central nervous system complications, and developed faster reactions to LOCMs. In particular, elderly patients older
Table 3 Time to onset of reaction and symptoms

\begin{tabular}{|c|c|c|c|}
\hline Variables & Moderate $(n=33)$ & Severe $(n=37)$ & $p$ \\
\hline Time to onset (min) & $7.0(4.0-19.5)$ & $4.0(2.5-5.0)$ & $<0.001$ \\
\hline Time to onset & & & 0.008 \\
\hline$<1 \mathrm{~h}$ (immediate) & $27(81.8)$ & $37(100.0)$ & \\
\hline$>1 \mathrm{~h}$ (delayed) & $6(18.2)$ & 0 & \\
\hline \multicolumn{4}{|l|}{ Symptoms } \\
\hline Skin/mucosal & $26(78.8)$ & $32(86.5)$ & 0.394 \\
\hline Urticaria & 24 & 26 & \\
\hline Angioedema & 21 & 16 & \\
\hline Respiratory & $19(57.6)$ & $16(43.2)$ & 0.231 \\
\hline Dyspnea & 12 & 14 & \\
\hline Neck tightness & 6 & 2 & \\
\hline Stridor, hoarseness & 3 & 1 & \\
\hline Hypoxia & 0 & 1 & \\
\hline Central nervous system & $9(27.3)$ & $25(67.6)$ & 0.001 \\
\hline Faint/dizziness & 7 & 18 & \\
\hline Loss of consciousness & 2 & 9 & \\
\hline Seizure & 0 & 4 & \\
\hline Headache & 0 & 1 & \\
\hline Cardiovascular & $8(24.2)$ & $36(97.3)$ & $<0.001$ \\
\hline Hypotension & 0 & 36 & \\
\hline Chest discomfort & 7 & 4 & \\
\hline Tachycardia & 1 & 1 & \\
\hline Gastrointestinal & $4(12.1)$ & $10(27.0)$ & 0.120 \\
\hline Vomiting & 3 & 8 & \\
\hline Abdominal pain & 1 & 4 & \\
\hline Diarrhea & 0 & 0 & \\
\hline
\end{tabular}

than 60 years and a time to reaction onset of less than 5 min were risk factors of a severe reaction.

\section{Risk factors of a severe adverse reaction to LOCMs}

Until recently, very few clinical studies have investigated the association between patient age and adverse reactions to contrast media. Shehadi et al. and Lieberman et al. demonstrated that adverse reactions occurred most frequently in patients in their 20 s and 50 s and least frequently in patients at either end of the age spectrum (Shehadi 1975). Furthermore, a recent study of gadobutrol safety in elderly patients demonstrated no greater incidence of adverse reactions in elderly patients ( $\geq 65$ years) compared with younger adults (Endrikat et al. 2015). By contrast, Cashman et al. suggested that the mortality was age-related and that the cutoff value for age was 65 years (Cashman et al. 1991). Similar results were obtained in the present study. We suggest that the discordant results stem from the different characteristics between LOCMs, high osmolality contrast media of CT tests, and gadobutrol used for magnetic resonance 
Table 4 Pretreatment, treatment, and outcome

\begin{tabular}{|c|c|c|c|}
\hline Variables & Moderate $(n=33)$ & Severe $(n=37)$ & $p$ \\
\hline Pretreatment & $10(30.3)$ & $18(48.6)$ & 0.118 \\
\hline Antihistamine & 10 & 18 & \\
\hline Hydrocortisone & 10 & 18 & \\
\hline \multicolumn{4}{|l|}{ Treatment } \\
\hline $\mathrm{H}_{1}$ blocker & $33(100.0)$ & 34 (91.9) & 0.242 \\
\hline $\mathrm{H}_{2}$ blocker & $23(69.7)$ & $23(62.2)$ & 0.507 \\
\hline$\beta_{2}$ agonist nebulizer & $2(6.1)$ & $2(5.4)$ & 1.000 \\
\hline Steroid & $25(75.8)$ & $32(86.5)$ & 0.249 \\
\hline Hydrocortisone & 16 & 22 & \\
\hline Methylprednisolone & 14 & 18 & \\
\hline Dexamethasone & 0 & 0 & \\
\hline Epinephrine & $11(33.3)$ & $37(100.0)$ & $<0.001$ \\
\hline Intramuscular & 5 & 28 & \\
\hline Subcutaneous & 3 & 3 & \\
\hline Intravenous & 3 & 6 & \\
\hline Disposition & & & 0.004 \\
\hline Discharge & $26(78.8)$ & $19(51.4)$ & \\
\hline Admission & $4(12.1)$ & $17(45.9)$ & \\
\hline AMA discharge & $3(9.1)$ & $1(2.7)$ & \\
\hline Death & 0 & 0 & \\
\hline
\end{tabular}

AMA against medical advice, $\beta^{2}$ beta receptor $2, H_{1}$ histamine receptor $1, H_{2}$ histamine receptor 2

imaging. Older patients presented a higher risk of a severe reaction, and the cutoff value for age was 60 years. The pulse rates of patients in the severe group were generally lower than those of patients in the moderate group (75 vs. 86, respectively), although this difference was not significant. These results suggest that hypotension in old age may be further complicated by failure of the heart to adequately compensate for vasodilatation.

To date, only a few studies have reported a relationship between an adverse reaction and the time to onset of the reaction, and the results have varied. Kunishima et al. reported that the symptoms of overall adverse reactions to contrast media occurred primarily within $5 \mathrm{~min}$ of administration of the contrast media (Kunishima et al. 2009). Hartman et al. and Shehadi found that moderate and severe reactions occurred within $20 \mathrm{~min}$ of administration (Hartman et al. 1982; Shehadi 1985). In the present study, the time to onset of reaction differed significantly between the moderate and severe groups. Patients with a severe reaction developed symptoms within $15 \mathrm{~min}$ of LOCM administration, and those with a moderate reaction developed symptoms within $72 \mathrm{~min}$. In addition, the cutoff value for the time to onset of reaction was $5 \mathrm{~min}$ for severe reactions. These findings suggest that it is necessary to observe patients for at least 15 min after they have undergone a CT examination with contrast media. Clinicians should monitor the occurrence of rapid deterioration, particularly if symptoms develop within 5 min of the administration of contrast media.

According to previous studies, risk factors associated with adverse reactions to contrast media are a history of allergy to foods, drugs, or contrast media and concurrent use of a beta-blocker. In the present study, we evaluated whether these risk factors could influence the severity of adverse reactions. In another study, the incidence of adverse reactions in patients with a previous allergy history was 4.5 times higher than that in patients with no allergy history (Davenport et al. 2009). However, the results of the present study demonstrated no statistical relationship between severity of an adverse reaction and previous allergy history to contrast media, foods, or medication.

\section{Effects of premedication and change in contrast media}

Pretreatment is not necessary in patients with no history of adverse reactions to contrast media (Dawson and Sidhu 1993; Radhakrishnan et al. 2005). According to Freed and Davenport, when a pretreated patient develops a breakthrough reaction, the severity of the reaction is similar to that of the adverse reaction previously experienced by the patient (Freed et al. 2001; Davenport et al. 2009). However, the results of the present investigation revealed no significant relationship between premedication and severity of adverse reactions. By contrast, a greater number of patients in the severe group were pretreated compared with the moderate group. We propose that our contrasting results are due to the inadequate dosage and frequency

Table 5 Logistic analysis for the prediction of severe adverse events in response to nonionic LOCMs

\begin{tabular}{llll}
\hline Variables & Univariate analysis, OR (95 \% Cl) & $\boldsymbol{p}$ & Multivariate analysis, OR (95 \% Cl) \\
\hline Age (years) & $1.049(1.005-1.096)$ & 0.029 & $1.053(1.002-1.106)$ \\
Sex (male) & $0.892(0.349-2.280)$ & 0.811 & 0.042 \\
Time to onset (min) & $0.797(0.666-0.954)$ & 0.013 & $0.005(0.671-0.966)$ \\
Previous allergy to LOCMs & $2.059(0.789-5.376)$ & 0.140 & 0.182 \\
Change of contrast & $0.512(0.191-1.369)$ & 0.121 & \\
Pretreatment & $2.179(0.815-5.825)$ & &
\end{tabular}

LOCM low osmolality contrast media, OR odds ratio 
in our protocol compared with the previous protocol. Thomsen et al. recommended the use of different contrast media in further studies investigating patients with a history of reactions to contrast media (Thomsen and Bush 1998). The results of the present study revealed a twofold decrease in the incidence of severe reactions when the contrast media administered to patients differed from those to which the patients showed a previous allergy, although this change was not statistically significant.

\section{Treatment of adverse reactions}

The ACR manual on contrast media recommends the use of antihistamines, steroids and epinephrine to treat moderate and severe reactions. In the present study, there was no significant difference in the number of patients who were treated with an antihistamine and a steroid in the moderate and severe groups. There was also no significant difference in the dosages of antihistamine between the two groups ( $1.36 \mathrm{vs.} 1.44, p=0.525$ ). Three patients in the severe group had only an epinephrine injection without an antihistamine and steroid, through a mistake of the medical team in the CT room. These patients showed improvement of symptoms after epinephrine administration; hence, the additional antihistamine and steroid were unnecessary in the ER. However, there was a large difference in the number of patients who were treated with epinephrine between the moderate and severe groups (33.3 vs. $100.0 \%$, relatively). Specifically, the use of epinephrine was more than twice as common in the severe group (6.1 vs. $45.9 \%, p<0.001$ ), and continuous intravenous administration of epinephrine was required for four patients in the severe group. The lower use of epinephrine in the moderate group than the severe group may be explained by the tendency of clinicians to hesitate when prescribing epinephrine to patients without significant hypotension and hypoxia. Through proper education, clinicians should be encouraged to increase the use of epinephrine for the treatment of moderate adverse reactions.

The limitations of this study are that it was designed retrospectively, data were collected from report forms and electronic medical records, and selection bias could not be eliminated. Another limitation is that patients who had mild reactions or who were not admitted to the ER were not enrolled in the study, rendering it impossible to evaluate the overall clinical characteristics of adverse reactions to nonionic LOCMs. Finally, a small sample size was assessed because the patient data were collected from a single hospital.

\section{Conclusion}

Compared with patients with a moderate reaction, those with a severe reaction were older, had higher blood pressures, showed more symptoms indicative of cardiovascular and central nervous system complications, and developed faster reactions to LOCMs. In particular, elderly patients older than 60 years and a time to reaction onset of less than 5 min were risk factors of a severe reaction.

\section{Authors' contributions}

SOH, DYK and YDS conceived of the study, collected the data, performed statistical analyses and prepared the first draft of the results, tables and manuscript. All authors read and approved the final manuscript.

\section{Author details}

${ }^{1}$ Department of Emergency Medicine, Hallym University Sacred Heart Hospital, Hallym University Medical Center, 22, Gwanpyeong-ro 170 Beon-gil, Dongan-gu, Anyang-si, Gyeonggi-do 431-070, Korea. ${ }^{2}$ Department of Emergency Medicine, University of Eulji College of Medicine and Eulji Medical Center, Seoul, Korea.

\section{Acknowledgements \\ None.}

\section{Competing interests}

The authors declare that they have no competing interests.

Received: 29 January 2016 Accepted: 20 May 2016

Published online: 30 June 2016

\section{References}

ACR Committee on Drugs and Contrast Media (2012) ACR manual on contrast media version. American College of Radiology, Reston, $\mathrm{p} 8$

ACR Committee on Drugs and Contrast Media (2013) ACR manual on contrast media version. American College of Radiology, Reston, p 9

Cashman JD, McCredie J, Henry DA (1991) Intravenous contrast media: use and associated mortality. Med J Aust 155:618-623

Christiansen C (2005) X-ray contrast media-an overview. Toxicology 209:185-187. doi:10.1016/j.tox.2004.12.020

Davenport MS, Cohan RH, Caoili EM, Ellis JH (2009) Repeat contrast medium reactions in premedicated patients: frequency and severity. Radiology 253:372-379. doi:10.1148/radiol.2532090465

Dawson P, Sidhu PS (1993) Is there a role for corticosteroid prophylaxis in patients at increased risk of adverse reactions to intravascular contrast agents? Clin Radiol 48:225-226. doi:10.1016/S0009-9260(05)80300-9

Endrikat J, Schwenke C, Prince MR (2015) Gadobutrol for contrast-enhanced magnetic resonance imaging in elderly patients: review of the safety profile from clinical trial, post-marketing surveillance, and pharmacovigilance data. Clin Radiol 70:743-751. doi:10.1016/j.crad.2015.03.011

Freed KS, Leder RA, Alexander C, DeLong DM, Kliewer MA (2001) Breakthrough adverse reactions to low-osmolar contrast media after steroid premedication. AJR Am J Roentgenol 176:1389-1392. doi:10.2214/ajr.176.6.1761389

Grant KL, Camamo JM (1997) Adverse events and cost savings three years after implementation of guidelines for outpatient contrast-agent use. Am J Health Syst Pharm 54:1395-1401

Hartman GW, Hattery RR, Witten DM, Williamson B Jr (1982) Mortality during excretory urography: Mayo Clinic experience. AJR Am J Roentgenol 139:919-922. doi:10.2214/ajr.139.5.919

Jacobs JE, Birnbaum BA, Langlotz CP (1998) Contrast media reactions and extravasation: relationship to intravenous injection rates. Radiology 209:411-416. doi:10.1148/radiology.209.2.9807567

Kunishima K, Takao H, Akahane M, Yoshioka N, Ohtomo K (2009) Safety of using iodized oil in chemoembolization for liver tumors in patients with previous adverse reactions to iodinated contrast media. Clin Imaging 33:365-368. doi:10.1016/j.clinimag.2008.12.009

Radhakrishnan S, Manoharan S, Fleet M (2005) Repeat survey of current practice regarding corticosteroid prophylaxis for patients at increased risk of adverse reaction to intravascular contrast agents. Clin Radiol 60:58-63. doi:10.1016/j.crad.2004.05.020 
Segal AJ, Bush WH Jr (2011) Avoidable errors in dealing with anaphylactoid reactions to iodinated contrast media. Invest Radiol 46:147-151. doi:10.1097/RLI.0b013e3181f5409e

Shehadi WH (1975) Adverse reactions to intravascularly administered contrast media. A comprehensive study based on a prospective survey. Am J Roentgenol Radium Ther Nucl Med 124:145-152. doi:10.2214/ ajr.124.1.145
Shehadi WH (1985) Death following intravascular administration of contrast media. Acta Radiol Diagn (Stockh) 26:457-461. doi:10.1177/028418518502600416

Thomsen HS, Bush WH Jr (1998) Adverse effects of contrast media: incidence, prevention and management. Drug Saf 19:313-324. doi:10.2165/00002018-199819040-00006

\section{Submit your manuscript to a SpringerOpen ${ }^{\circ}$ journal and benefit from:}

- Convenient online submission

- Rigorous peer review

- Immediate publication on acceptance

- Open access: articles freely available online

- High visibility within the field

- Retaining the copyright to your article 